\title{
CHILD syndrome: successful treatment of skin lesions with topical lovastatin and cholesterol lotion*
}

\author{
Karina Romero Sandoval ${ }^{1}$, Maria Cecília Rivitti Machado ${ }^{2}$, Zilda Najjar Prado de Oliveira², \\ Marcello Menta Simonsen Nico ${ }^{1}$
}

DOI: http:/ /dx.doi.org/10.1590/abd1806-4841.20198789

\begin{abstract}
CHILD syndrome (Congenital Hemidysplasia, Ichthyosiform erythroderma, Limb Defects) is a rare X-linked dominant disease. The authors report a 2-month-old patient presenting with typical features of CHILD syndrome that was treated with a topical solution containing cholesterol and lovastatin, with complete clearance of her CHILD nevus. The changes in skin lipid metabolism that explain the CHILD ichthyosiform nevus and their correction through topical application of cholesterol and lovastatin are discussed.
\end{abstract}

Keywords: Cholesterol; Ichthyosis; Lovastatin

\section{INTRODUCTION}

CHILD syndrome is a rare $\mathrm{X}$-linked dominant disease, of which about 100 cases have been reported. ${ }^{1}$ The term CHILD is an acronym for Congenital Hemidysplasia, Ichthyosiform erythroderma, and Limb Defects. ${ }^{2}$

CHILD syndrome results from mutations in the $\mathrm{NAD}(\mathrm{P}) \mathrm{H}$ steroid dehydrogenase-like protein) (NSDHL) gene leading to inhibition of cholesterol synthesis. ${ }^{3}$ This gene encodes a member of the enzyme complex that removes the C-4 methyl group from lanosterol. Mutations in the NSDHL gene prevent the post-lanosterol generation of cholesterol but concurrently lead to accumulation of upstream, potentially toxic metabolite. ${ }^{1}$
Most patients are female. The CHILD nevus is unilateral, follows Blaschko's lines, and may show ptychotropism (affinity for skin folds). ${ }^{1}$ Ipsilateral involvement of visceral structures may be rarely seen. ${ }^{1}$

Empirical therapies (keratolytics, emollients, corticosteroids, or cryotherapy) give poor results, as do systemic therapies such as oral retinoids and methotrexate. ${ }^{4}$ Surgical procedures such as dermabrasion or skin grafts can be tried. ${ }^{5}$

A pathogenesis-based therapy aiming at the disrupted cholesterol metabolism has been recently described with effective results. ${ }^{6}$ Topical application of cholesterol in combination with a

Received 12 September 2018.

Accepted 8 November 2018.

* Study conducted at the Division of Dermatology, Hospital das Clínicas, Faculdade de Medicina, Universidade de São Paulo, São Paulo, SP, Brazil. Financial support: None. Conflict of Interest: None.

1 Department of Dermatology, Hospital das Clínica, Faculdade de Medicina, Universidade de São Paulo, São Paulo, SP, Brazil.

2 Division of Dermatology, Ambulatório de Dermatologia Pediátrica, Hospital das Clínicas, Faculdade de Medicina, Universidade de São Paulo, São Paulo, SP, Brazil.

MaILING AdDREss:

Marcello Menta Simonsen Nico

E-mail: mentanico@hotmail.com

(C)2019 by Anais Brasileiros de Dermatologia 
cholesterol-synthesis inhibiting agent (statin) has the potential to reverse skin symptoms in CHILD syndrome by providing functional cholesterol while at the same time inhibiting the accumulation of toxic metabolites. ${ }^{6}$ Less than ten patients submitted to this treatment have been reported, none of them in Brazil. ${ }^{6}$

\section{CASE REPORT}

A 2-month-old female patient presented with features of CHILD syndrome. Findings included marked hypoplasia of the lower left limb as well as well-demarcated segmental erythematous and scaly plaques following Blaschko's lines, with sharp demarcation at the midline (Figure 1A). Radiologic studies of the affected limb showed hypoplasia of the fibula, tibia, tarsal, metatarsal, and phalangeal bones.

A topical 2\% lovastatin and 2\% cholesterol lotion was applied twice daily, and significant improvement was observed at the second week. After eight weeks the lesions were totally cleared; she remained well with thrice-a-week applications (Figure 1B-D; Figure 2).

\section{DISCUSSION}

Two mechanisms explain the cutaneous manifestations of CHILD syndrome: the cholesterol deficiency itself, which probably influences the proper formation of the corneocyte membrane, and the accumulation of toxic steroid precursors. ${ }^{6,7}$

It has been shown that cholesterol and its precursors play an important role in the regulation of the sonic hedgehog signaling pathway, causing the skeletal deformations in CHILD syndrome. ${ }^{8}$ Alterations in the cholesterol metabolism also results in defects in the lipid envelope of the stratum corneum (SC), leading to higher permeability to water and ions, increasing the $\mathrm{pH}$ and activating proteases that degrade corneodesmosomes and lipid processing enzymes for assembling the SC lipid envelope. The increased SC permeability also activates pro-inflammatory cytokines, which induce keratinocyte proliferation and differentiation abnormalities, leading to ichthyosis. Sterol precursors also accelerate degradation of 3-hydroxy-3-methylglutaryl coenzyme A reductase, which is the rate-limiting enzyme for cholesterol synthesis. ${ }^{1-3}$
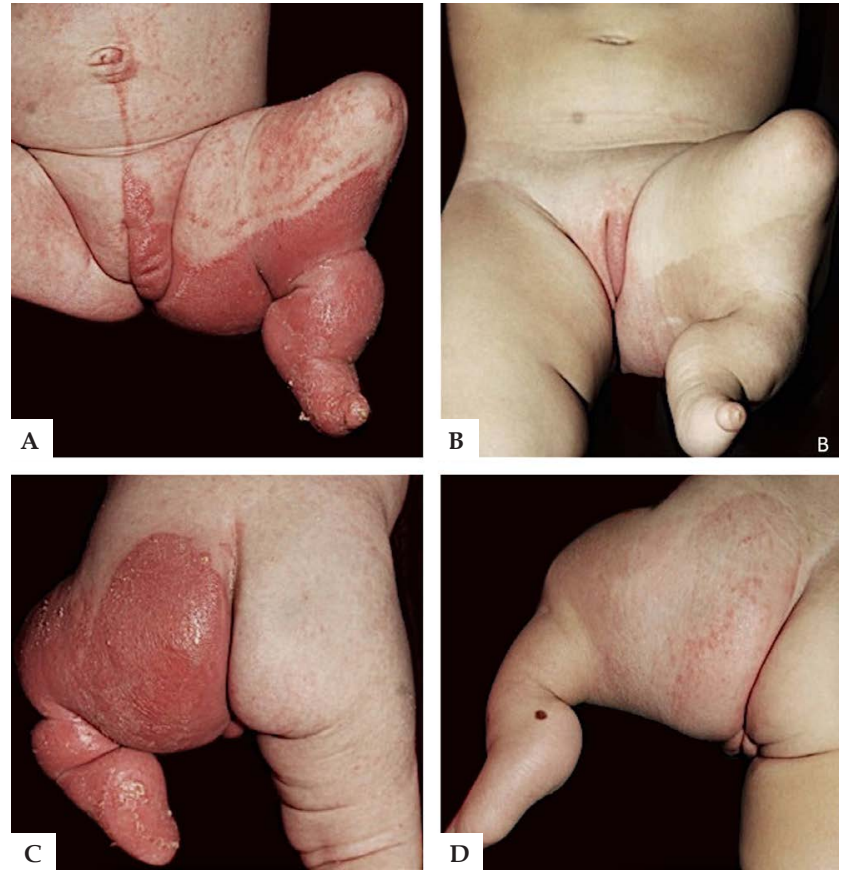

Figure 2: Lower limb and genital lesions before and during treatment - four-month follow-up

Thus, the replacement of cholesterol alone is not effective without the blockade of metabolite production, as has been observed. ${ }^{6}$ Nonetheless, association of cholesterol with lovastatin resulted in improvement. Other authors used $2 \%$ simvastatin instead of lovastatin. ${ }^{9}$

Animal studies have shown that transdermal application of lovastatin results in higher serum concentrations than those observed with oral administration, suggesting that the best route of administration for these drugs is transdermal. ${ }^{10}$

Further research on lipid metabolism of the skin might lead to more effective treatments of CHILD syndrome and other keratinization disorders. $\square$
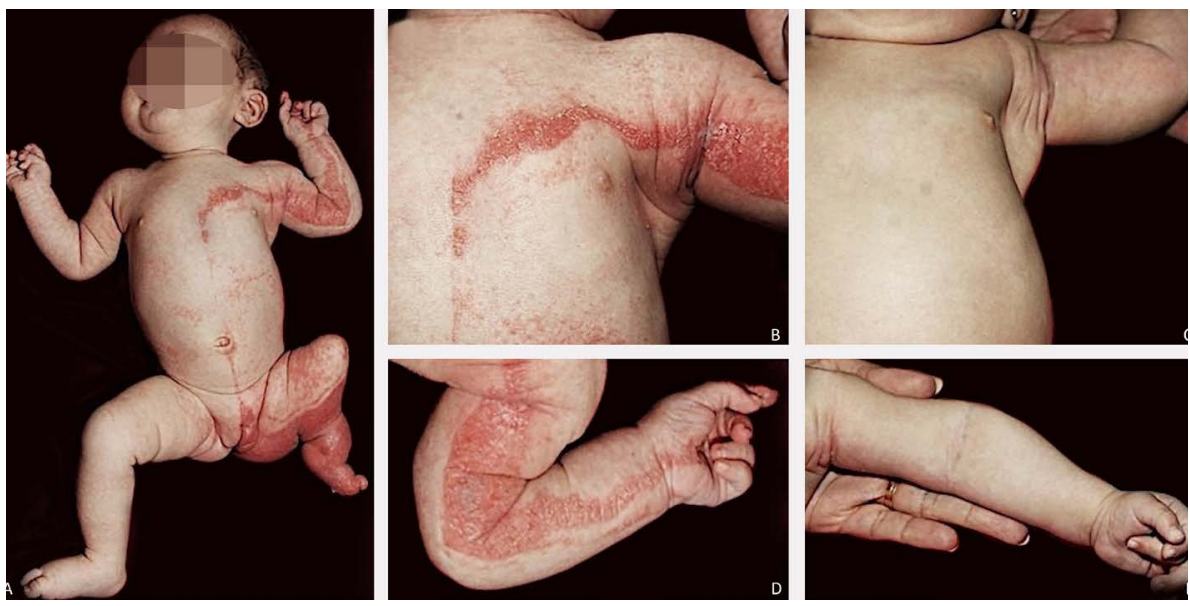

Figure 1: A - CHILD syndrome: typical unilateral segmentary lesion with lower limb hypoplasia. B, C, D, and E - Trunk and upper limb lesions before and during treatment 


\section{REFERENCES}

1. Lelonek E, Matusiak $\measuredangle$, Bieniek A, Szepietowski JC.. Unilateral erythematous lesions with wax-like scaling and limb abnormalities: a quiz. Acta Derm Venereol. 2016;961004-8.

2. Happle R, Koch H, Lenz W. The CHILD syndrome: congenital hemidysplasia with ichtyosiform erythroderma and limd defects. Eur J Pediatr. 1980;134:27-33.

3. Porter FD, Herman GE. Malformation syndromes caused by disorders of cholesterol synthesis. J Lipid Res. 2011;52:6-34.

4. Hebert AA, Esterly NB, Holbrook KA, Hall JC. The CHILD syndrome. Histologic and ultrastructural studies. Arch Dermatol. 1987;123:503-9.

5. Fink-Puches R, Soyer HP, Pierer G, Kerl H, Happle R. Surgical treatment of CHILD nevus. Eur J Dermatol. 2000;10:262-4.

6. Paller AS, van Steensel MA, Rodriguez-Martín M, Sorrell J, Heath C, Crumrine D, et al. Pathogenesis-based therapy reverses cutaneous abnormalities in an inherited disorder of distal cholesterol metabolism. J Invest Dermatol. 2011:131:2242-8.
7. Christiansen AG, Koppelhus U, Sommerlund M. Skin Abnormalities in CHILD syndrome successfully treated with pathogenesis-based therapy. Acta Derm Venereol. 2015;95:752-3.

8. Stottmann RW, Turbe-Doan A, Tran P, Kratz LE, Moran JL, Kelley RI, et al. Cholesterol metabolism is required for intracellular hedgehog signal transduction in vivo. PLoS Genet. 2011;7:e1002224.

9. Alexopoulos A, Kakourou T. CHILD syndrome: successful treatment of skin lesions with topical simvastatin/cholesterol ointment $\mathrm{C}$ a case report. Pediatr Dermatol. 2015;32:e145-7.

10. Gutierrez GE, Lalka D, Garrett IR, Rossini G, Mundy GR. Transdermal application of lovastatin to rats causes profound increases in bone formation and plasma concentrations. Osteoporos Int. 2006:17:1033-42.

\section{AUTHORS'CONTRIBUTIONS}

\begin{tabular}{|c|c|c|}
\hline Karina Romero Sandoval & (iD) ORCID & 0000-0001-7671-5019 \\
\hline \multicolumn{3}{|c|}{ Elaboration and writing of the manuscript } \\
\hline Maria Cecília Rivitti Machado & (iD) ORCID & 0000-0003-2910-7330 \\
\hline \multicolumn{3}{|c|}{ Elaboration and writing of the manuscript } \\
\hline Zilda Najjar Prado de Oliveira & (iD) ORCID & 0000-0002-8596-1999 \\
\hline \multicolumn{3}{|c|}{ Effective participation in research orientation } \\
\hline Marcello Menta Simonsen Nico & (iD) $O R C I D$ & 0000-0001-7968-0624 \\
\hline
\end{tabular}

Elaboration and writing of the manuscript; Effective participation in research orientation; Intellectual participation in propaedeutic and/or therapeutic conduct of the cases studied

How to cite this article: Sandoval KR, Machado MCR, Oliveira ZNP, Nico M. CHILD syndrome: successful treatment of skin lesions with topical lovastatin and cholesterol lotion. An Bras Dermatol. 2019;94(3):341-3. 